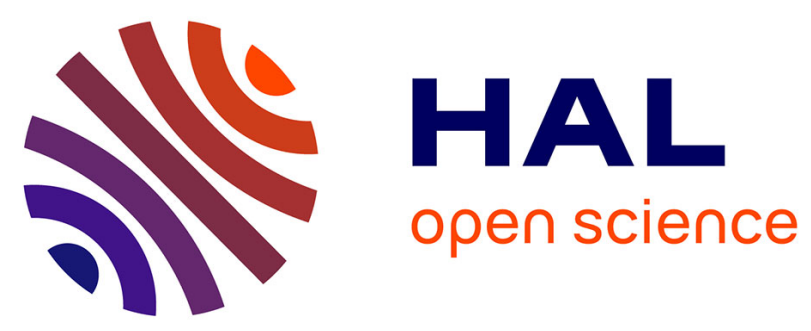

\title{
Quadrastichus erythrinae Kim: un redoutable ravageur pour les érythrines de Guadeloupe et de Martinique (Hymenoptera, Eulophidae, Tetrastichinae)
}

\author{
Jean Etienne, Eddy Dumbardon-Martial
}

\section{- To cite this version:}

Jean Etienne, Eddy Dumbardon-Martial. Quadrastichus erythrinae Kim : un redoutable ravageur pour les érythrines de Guadeloupe et de Martinique (Hymenoptera, Eulophidae, Tetrastichinae). Bulletin de la Société Entomologique de France, 2013, 118 (2), pp.155-158. hal-02649453

\section{HAL Id: hal-02649453 \\ https://hal.inrae.fr/hal-02649453}

Submitted on 29 May 2020

HAL is a multi-disciplinary open access archive for the deposit and dissemination of scientific research documents, whether they are published or not. The documents may come from teaching and research institutions in France or abroad, or from public or private research centers.
L'archive ouverte pluridisciplinaire HAL, est destinée au dépôt et à la diffusion de documents scientifiques de niveau recherche, publiés ou non, émanant des établissements d'enseignement et de recherche français ou étrangers, des laboratoires publics ou privés. 


\title{
Quadrastichus erythrinae Kim : un redoutable ravageur pour les érythrines de Guadeloupe et de Martinique (Hymenoptera, Eulophidae, Tetrastichinae)
}

\author{
par Jean ETIEnNE* \& Eddy Dumbardon-MarTial** \\ * INRA, centre Antilles-Guyane, Domaine Duclos, F - 97170 Petit-Bourg, Guadeloupe <jean.etienne2@wanadoo.fr> \\ ** FREDON-Martinique, Croix-Rivail, F-97224 Ducos <eddy.dumbardon@wanadoo.fr>
}

Résumé. - Quadrastichus erythrinae Kim, 2004, est signalé pour la première fois en Guadeloupe et en Martinique. Les dégâts sont considérables principalement sur Erythrina variegata var. fastigiata introduit il y a une trentaine d'années dans ces deux îles pour l'établissement des haies de protection en zones bananières. Les études menées à Hawaii ont montré que seule la lutte biologique pouvait contrôler cet Eulophidae. Dans l'immédiat la réutilisation de Dracaena fragrans en remplacement des érythrines est préconisée pour la mise en place des nouvelles haies.

Abstract. - Quadrastichus erythrinae Kim: a formidable pest for erythrina in Guadeloupe and Martinique (Hymenoptera, Eulophidae, Tetrastichinae). Quadrastichus erythrinae Kim, 2004, is reported for the first time in Guadeloupe and Martinique. Damages are particularly considerable to Erythrina variegata var. fastigiata introduced 30 years ago on these two Islands for the establishment of protection hedges in banana zone. The studies led to Hawaii showed that only the biological control could control this Eulophidae. At this time, replacing Erythrina by Dracaena fragrans is recommended for the establishment of new hedgerows.

Keywords. - Eulophidae, Erythrina gall wasp, Guadeloupe, Martinique, Caribbean region.

Quadrastichus erythrinae Kim, 2004, a été décrit à partir de spécimens en provenance de Singapour, de l'île Maurice et de l'île de la Réunion (Кім et al., 2004). Cette espèce inféodée au genre Erythrina L. (Fabaceae) est particulièrement invasive puisque depuis sa description elle aurait pratiquement fait le tour du monde ! Il est possible aussi que, présente un peu partout, elle n'ait pas été détectée et soit passée inaperçue. Quoiqu'il en soit elle est signalée actuellement de Hawaii, Taiwan, Hong Kong, Chine, Inde, Thaïlande, Samoa américaines, Guam, Okinawa, Japon (LA SALle et al., 2009). Dans la région néotropicale elle a été signalée pour la première fois en Floride (Etats-Unis) par Wiley \& Skelley en 2006. Des études récentes ont montré que dans sa région d'origine, l'Afrique de l'Est, cette espèce est limitée par divers parasitoïdes (Prinsloo \& Kelly, 2009) dont certains sont considérés comme de bons candidats à la lutte biologique (GATes \& Delvare, 2008 ; LA SAlle et al., 2009).

Les premières découvertes de Q. erythrinae dans les Antilles françaises ont été faites en Martinique à Fort-de-France le 4.V.2012 (E. D.-M.), puis en Guadeloupe dans la commune de Baie-Mahault, section de Daubin le 6.V.2012 (J. E.), dans des haies d'érythrines.

Erythrines attaquées. - Les érythrines utilisées comme brise-vent ont été identifiées dans les deux îles comme étant Erythrina variegata var. fastigiata (Guillaumin), correspondant vraisemblablement au "Peuplier canaque" de Nouvelle-Calédonie (Fournet, 2002) (D. Marival, comm. pers.). Très commune dans nos îles, cette espèce a été introduite il y a environ une trentaine d'années pour remplacer les Dracaena fragrans (L.) Ker-Gawl (Dracaenaceae) utilisés alors pour l'établissement de haies. Aujourd'hui l'érythrine est largement répartie, notamment en zones bananières, en raison de sa croissance rapide qui permet de constituer des haies brisevent qui sont de véritables rideaux protecteurs de plus de $10 \mathrm{~m}$ de hauteur. Elle occupe aussi 
la même fonction dans les jardins privés et publics. En dehors de cette variété, ce sont les arbres d'ornement Erythrina variegata var. orientalis (L.) Merr et Erythrina variegata var. variegata L. qui sont également fortement attaqués. Il est intéressant de constater qu'Erythrina corallodendron var. corallodendron L., espèce endémique des Antilles et devenue pantropicale, n'a pas fait l'objet jusqu'à présent d'attaques de Quadrastichus erythrinae. Cette Erythrine était utilisée autrefois en Guadeloupe pour le bornage des parcelles à cause de sa rusticité et sa grande longévité.

Dégâts. - Les larves de Quadrastichus erythrinae se développent à l'intérieur des tissus végétaux des érythrines attaquées. Elles induisent des galles caractéristiques sur les feuilles,
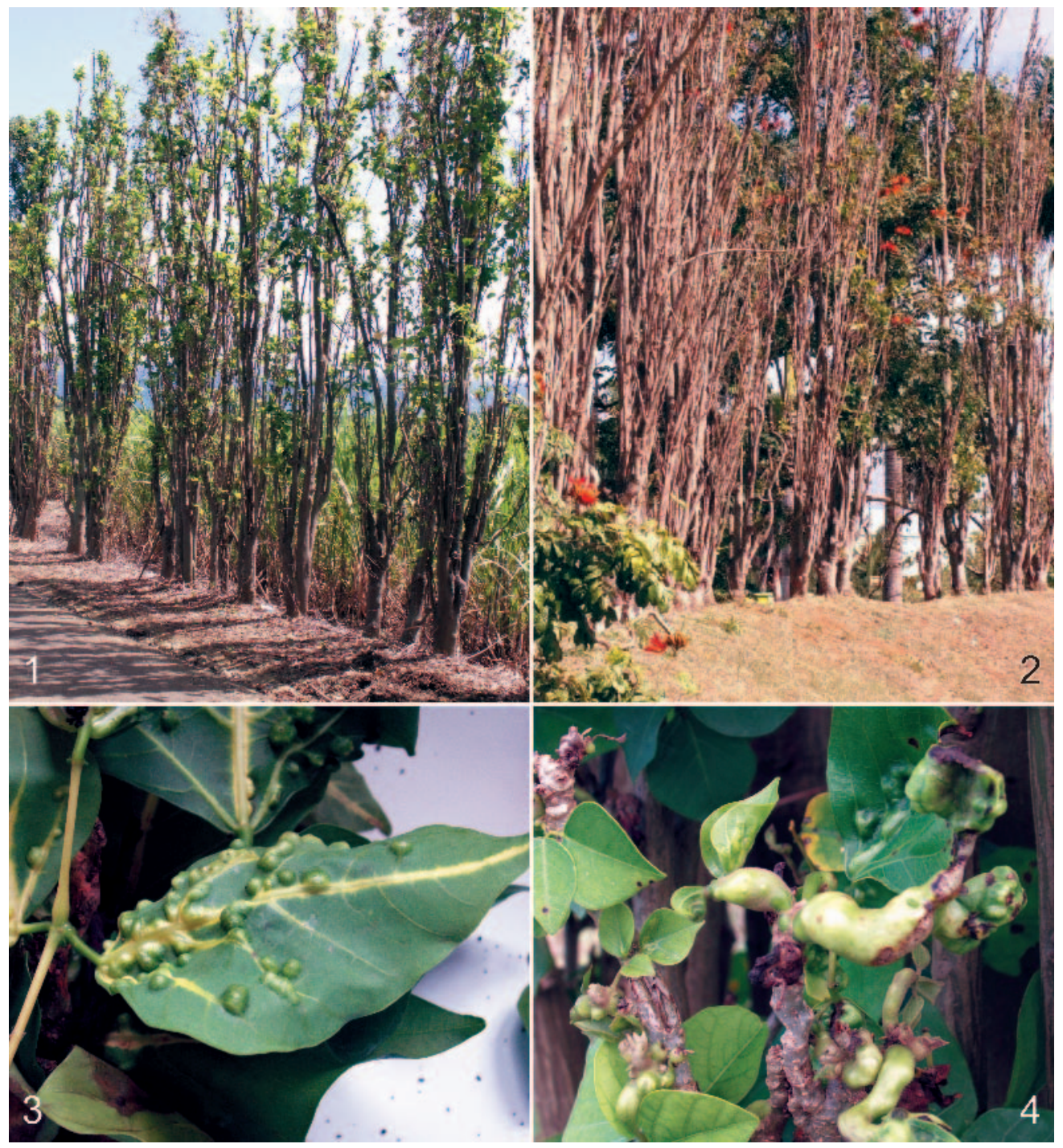

Fig. 1-4. - 1, Dépérissement d'une haie d'érythrines. - 2, Mortalité d'érythrines. - 3, Galles de Quadrastichus erythrinae Kim sur feuille d'érythrine. -4, Déformations provoquées par Q. erythrinae. 
les pétioles, les tiges et provoquent des déformations spectaculaires des repousses. Les dégâts sont considérables, et les arbres attaqués présentent une masse végétative rabougrie puis finissent le plus souvent par mourir.

En Guadeloupe et en Martinique, les dégâts sont présents sur toutes les érythrines brisevent où qu'elles soient. Au regard de la situation sanitaire actuelle, il est fort probable que ces haies soient à terme condamnées. En effet, un bon nombre de celles-ci sont déjà mortes [souvent couvertes de lianes d'Ipomoea sp. (Convolvulaceae)], la plupart sont en train de dépérir ou sont déjà en grande partie fortement contaminées. Curieusement ces dégâts semblent ne pas avoir inquiété pour l'instant les professionnels (agriculteurs et agents d'espaces verts) et les jardiniers amateurs. Cela explique probablement que ce nouveau ravageur n'ait été mis en évidence que dernièrement alors qu'il est très certainement présent dans nos deux îles depuis déjà un bon nombre d'années.

Moyens de lutte. - En Guadeloupe et en Martinique la lutte mécanique par la taille sévère, l'arrachage des arbres morts et la replantation apparaissent inutiles. Les repousses sont immédiatement attaquées et les nouvelles plantations le sont également. A Hawaii, cette méthode de lutte ainsi que la lutte chimique se sont avérées totalement inefficaces et ont été très vite abandonnées au profit de la lutte biologique (RAMADAN, 2007). En effet, après la détection de Quadrastichus erythrinae en 2005, les professionnels hawaiiens ont procédé à l'introduction et à des tests de spécificité d'un Hyménoptère parasitoïde de la famille des Eurytomidae : Eurytoma erythrinae Gates \& Delvare, 2008. Les tests ayant été satisfaisants, E. erythrinae a fait l'objet de lâchers en 2008 dans tout l'archipel et l'efficacité de son action biologique a pu être constatée dès 2010 (Kaufman et al., 2010 ; Meyer, 2010).

Par conséquent, en Guadeloupe et en Martinique, l'amélioration de l'état sanitaire des érythrines devrait se faire rapidement sur la base d'une lutte biologique impliquant un agent régulateur efficace. L'exemple des Cycas dans ces deux îles a bien montré que faute d'avoir pu introduire les auxiliaires disponibles en Floride, la disparition de cette plante a été inévitable (ETIENNE, 2007).

Dans l'immédiat, la constitution des nouvelles haies brise-vent ne pourra plus s'effectuer à partir des érythrines jusqu'alors utilisées. Dans ces conditions le recours, à nouveau, au Dracaena fragrans paraît la solution la plus facile à mettre en œuvre en attendant que des expérimentations puissent mettre en évidence d'autres espèces végétales plus prometteuses.

REMERCIEMENTS. - Nous remercions tout particulièrement notre ami et collègue Gérard Delvare (CIRAD Montpellier) qui a bien voulu examiner nos échantillons de Quadrastichus et confirmer nos identifications. Nous exprimons également nos vifs remerciements à nos collègues Jacques Fournet et Daniel Marival (INRA, AntillesGuyane) pour l'actualisation des noms de certaines érythrines.

\section{AUTEURS CITÉS}

ETIEnNe J., 2007. - Pour la sauvegarde de Cycas en Guadeloupe. L'Entomologiste, 63 (5) : 271-275.

Fournet J., 2002. - Flore illustrée des phanérogames de Guadeloupe et de Martinique. Nouvelle édition. Cirad \& Gondwana éditions, 2 tomes : $2538 \mathrm{p}$.

Gates M. \& Delvare G., 2008. - A new species of Eurytoma (Hymenoptera: Eurytomidae) attacking Quadrastichus spp. (Hymenoptera: Eulophidae) galling Erythrina spp. (Fabaceae), with a summary of African Eurytoma biology and species checklist. Zootaxa, 1751 : 1-24.

Kaufman L., Wright M., Messing R., Rubinoff D., Yalemar J. \& Nagamine W., 2010. - Rapid Global Invasion by Quadrastichus erythrinae (Eulophidae), the Erythrina Gall Wasp and the Hawaii Biological Control Success. Department of Plant and Environmental Protection Sciences, University of Hawaii, 36 p. 
Kim I. K., Delvare G. \& La Salle J., 2004. - A new species of Quadrastichus (Hymenoptera: Eulophidae): A Gall-inducing Pest on Erythrina (Fabaceae). Journal of Hymenoptera Research, 13 : 243-249.

La Salle J., Ramadan M. \& Kumashiro B. R., 2009. - A new parasitoid of the Erythrina Gall Wasp, Quadrastichus erythrinae Kim (Hymenoptera: Eulophidae). Zootaxa, 2083 : 19-26.

MEYER J.-Y., 2010. - L'introduction et l'invasion récente de Quadrastichus erythrinae, insecte parasite des Erythrines, à Tahiti : impacts et solutions ? Comité de Lutte Espèces envahissantes, 29 septembre 2010, Papeete, Tahiti : 8 p.

Prinsloo G. L. \& Kelly J. A., 2009. - The tetrastichine wasps (Hymenoptera: Chalcidoidea: Eulophidae) associated with galls on Erythrina species (Fabaceae) in South Africa, with description of five new species. Zootaxa, $2083: 27-45$.

RAmadan M. M., 2007. - A survey in Africa for potential biocontrol agents of the Erythrina gall wasp, Quadrastichus erythrinae, a new invasive species in the Pacific Region. Journal of Insect Science, 7 (16) : 19-20.

Wiley J. \& Skelley P., 2006. - Erythrina Gall Wasp, Quadrastichus erythrinae Kim, in Florida. Pest Alerts-Erythrina Gall Wasp in FL, DPI- FDACS, 3 p. 